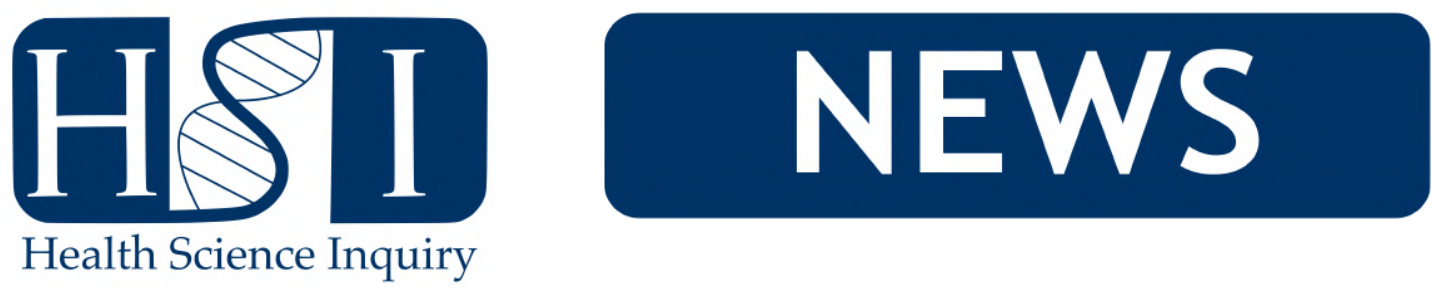

\title{
Microbes on the Mind: The Promise of Psychoactive Probiotics to Treat Mental IIIness
}

\section{Kevin Champagne-Jorgensen}

The development of novel pharmaceuticals for psychiatric illnesses has stagnated throughout the past few decades, leaving many scientists searching for alternative treatment strategies [1]. One promising avenue is the use of certain live microorganisms as oral supplements (termed probiotics).

This isn't as far-fetched as it may seem. We've known for decades that the trillions of microorganisms that inhabit our gastrointestinal tract (known as our gut microbiota) have diverse roles in modulating our physiology, including gastrointestinal and immune function [2]. But recently there's been a surge of research demonstrating that these microbes may also have important roles in brain function and development. Numerous clinical studies have found abnormal gut microbiota diversity in patients with some psychiatric or neurological illnesses, including depression, autism, and Alzheimer's disease [3]. Exploratory studies have further shown that animals without microbes develop abnormal behaviour and brain structure, while normal animals fed with specific bacteria or microbial communities can develop predictably altered behaviour [3].

To get an expert's take on this topic we spoke with Dr. Thomas Tompkins, research director at Lallemand Health Solutions in Montreal and a leader of research in this area. Tompkins noted that, while the idea of treating mental illness with live bacteria had been proposed as early as 1910, it was obscure, and only first occurred to his team in the early 2000 s [4]. This is when, according to Tompkins, they noted that in previous unrelated clinical studies, "participants reported that when they took the probiotics, they were getting better sleep and feeling less aggravated" [4]. These observations prompted a series of exploratory studies in both rodents and humans, which consistently suggested reduced anxiety-, depression-, and stress-related symptoms after treatment with Lallemand Health Solution's Probio'Stick probiotic (a combination of the bacteria Lactobacillus helveticus Rosell-52 and Bifidobacterium longum Rosell-175) [4]. While they are promising, these studies were early-phase and relatively small. But as Tompkins explained, we shouldn't have to wait long; more clinical work is set to be published over the next couple of years, and his colleagues are currently conducting a larger clinical trial of Probio'Stick in participants with major depressive disorder [4].

While the work of Tompkins and his colleagues is a good example of a promising probiotic showing beneficial psychoactive effects in human patients, it is unfortunately one of few. The majority of the work in this area is preclinical, where investigators are using animal models to uncover mechanisms that may allow microbes to affect the brain.

One of the pioneering laboratories in this field is that of Dr. John Bienenstock, director of the Brain-Body Institute and distinguished university professor at McMaster University. Bienenstock and his colleagues are actively researching a number of microbial strains to determine the mechanisms underlying their psychoactivity. A major focus of Bienenstock's research is the bacterium Lactobacillus rhamnosus JB-1, which has consistently decreased anxiety-like, depressive-like, and stress-related behaviours in rodent models [5]. His team and others have shown that some bacteria (such as $L$. rhamnosus JB-1) can produce neuroactive compounds, including neurotransmitters or short chain fatty acids. Moreover, he and his colleagues also demonstrated that some neuroactive properties of particular bacteria are dependent on the vagus nerve (a major neural connection between the brain and the gastrointestinal tract) [5]. As Bienenstock explained, there are many different pathways by which gut microbes may interact with the nervous system, including directly in the gut, neuronally through gut-connected nerves, and systemically via the immune or circulatory systems [5].

Though progress thus far is encouraging, both Tompkins and Bienenstock agreed that this research has many limitations. For example, Tompkins indicated that probiotic activity may depend on its location in the gut, which may limit their efficacy [4]. However, he believes that this and other issues may well be overcome by the use of genetic engineering; as Tompkins put it, "if we can find specific pathways to target... we could alter a microbe to deliver a very specific compound to a specific region of the gut where its receptors for uptake are". Furthermore, according to Bienenstock, mounting evidence suggests that other members of the gut microbiota play important roles in modulating the efficacy of probiotics, and that some probiotic activity may in fact be mediated by as-of-yet-overlooked components [5]. As he told us, "the whole question of non-bacterial microbes, such as the bacteriophages, other viruses, archaea, and yeasts that 
also populate our intestines, is only at the very beginning of understanding and testing, and it's very limiting to simply assume that the only thing that's important is bacteria."

Thus, while much of the research in this area remains preliminary, both experts we spoke to are confident that it will progress fruitfully. The next few years should see an increasing number of probiotics entering clinical trials for their psychoactive properties, and some may even reach the market in the coming decades. Where future research will take this field is certainly up for debate, but because of the substantial scientific and commercial interest, strong preclinical promise, and potential for seminal discoveries in this area, we can be certain of years of exciting discoveries to come.

\section{REFERENCES}

[1] Friedman RA. A Dry Pipeline for Psychiatric Drugs. The New York Times; 2013. Available from: https://www. nytimes.com/2013/08/20/health/a-dry-pipeline-for-psychiatric-drugs.html.

[2] Schroeder BO, Bäckhed F. Signals from the gut microbiota to distant organs in physiology and disease. Nature medicine. 2016;22(10):1079.

[3] Sarkar A, Harty S, Lehto SM, Moeller AH, Dinan TG, Dunbar RI, et al. The microbiome in psychology and cognitive neuroscience. Trends in cognitive sciences. 2018;22(7):611-636.

[4] Tompkins T. Interview; 11 December 2018.

[5] Bienenstock J. Interview; 07 December 2018.

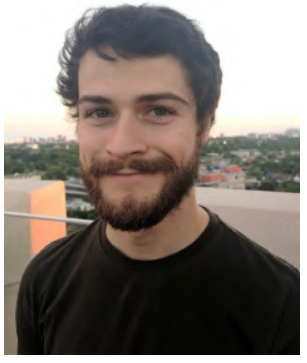

Kevin completed his HBSc in Biopsychology at the University of Winnipeg in 2015, and is now a PhD candidate in the Neuroscience Graduate Program at McMaster University. His research focuses on mechanisms of communication between gut microbes and the nervous and immune systems of their host. When not in the lab, Kevin enjoys rock climbing and complaining about Hamilton's weather. 